Louisiana State University

LSU Digital Commons

7-1-2005

\title{
Accessing properties of electron wave packets generated by attosecond pulse trains through time-dependent calculations
}

Johan Mauritsson

Louisiana State University

Mette B. Gaarde

Louisiana State University

Kenneth J. Schafer

Louisiana State University

Follow this and additional works at: https://digitalcommons.Isu.edu/physics_astronomy_pubs

\section{Recommended Citation}

Mauritsson, J., Gaarde, M., \& Schafer, K. (2005). Accessing properties of electron wave packets generated by attosecond pulse trains through time-dependent calculations. Physical Review A - Atomic, Molecular, and Optical Physics, 72 (1) https://doi.org/10.1103/PhysRevA.72.013401 


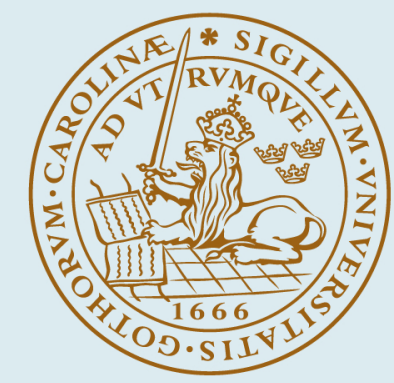

\title{
LUND UNIVERSITY
}

\section{Accessing properties of electron wave packets generated by attosecond pulse trains through time-dependent calculations}

\author{
Mauritsson, Johan; Gaarde, Mette; Schafer, Kenneth J \\ Published in: \\ Physical Review A (Atomic, Molecular and Optical Physics) \\ DOI: \\ 10.1103/PhysRevA.72.013401
}

2005

Link to publication

Citation for published version (APA):

Mauritsson, J., Gaarde, M., \& Schafer, K. J. (2005). Accessing properties of electron wave packets generated by attosecond pulse trains through time-dependent calculations. Physical Review A (Atomic, Molecular and Optical Physics), 72(1). https://doi.org/10.1103/PhysRevA.72.013401

Total number of authors:

3

\footnotetext{
General rights

Unless other specific re-use rights are stated the following general rights apply:

Copyright and moral rights for the publications made accessible in the public portal are retained by the authors and/or other copyright owners and it is a condition of accessing publications that users recognise and abide by the legal requirements associated with these rights.

- Users may download and print one copy of any publication from the public portal for the purpose of private study or research.

- You may not further distribute the material or use it for any profit-making activity or commercial gain

- You may freely distribute the URL identifying the publication in the public portal

Read more about Creative commons licenses: https://creativecommons.org/licenses/

Take down policy

If you believe that this document breaches copyright please contact us providing details, and we will remove access to the work immediately and investigate your claim.
} 


\title{
Accessing properties of electron wave packets generated by attosecond pulse trains through time-dependent calculations
}

\author{
Johan Mauritsson, Mette B. Gaarde, and Kenneth J. Schafer \\ Department of Physics and Astronomy, Louisiana State University, Baton Rouge, Louisiana 70803-4001, USA
}

(Received 2 March 2005; published 1 July 2005)

\begin{abstract}
We present a time-dependent method for calculating the energy-dependent atomic dipole phase that an electron acquires when it is ionized by the absorption of a single ultraviolet photon. Our approach exactly mirrors the method used to experimentally characterize a train of attosecond pulses. In both methods the total electron phase is measured (calculated) via a two-photon interference involving the absorption or emission of an additional infrared photon in the continuum. In our calculation we use a perfect (zero spectral phase) light field and so extract the atomic dipole phase directly from the electron wave packet. We calculate the atomic phase for argon, neon, and helium at low infrared intensities and compare them to previous perturbative calculations. At moderate infrared probe intensities, we find that that the dipole phase can still be reliably determined using two-photon interference, even when higher-order processes are non-negligible. We also show that a continuum structure, in this case a Cooper minimum in argon, significantly affects the probability for infrared absorption and emission over a range of energies around the minimum, even at low infrared intensities. We conclude that well-characterized attosecond pulse trains can be used to examine continuum structures in atoms and molecules.
\end{abstract}

DOI: 10.1103/PhysRevA.72.013401

PACS number(s): $32.80 . \mathrm{Rm}, 42.65 . \mathrm{Ky}$

\section{INTRODUCTION}

Attosecond pulse trains (APTs) are currently produced and characterized on a regular basis in many laboratories [1-8]. The attosecond pulses are synthesized in the extreme ultraviolet (XUV) wavelength region from high-order harmonics generated in the interaction between a gas and an intense infrared (IR) laser pulse [9]. The complete reconstruction of an APT requires knowledge of both the spectral amplitude and phase of all the frequency components (harmonics) used to synthesize the train. Though the amplitudes are easily measured, the spectral phases are not. The method most commonly used to characterize APTs is therefore based on a two-step process. First, the individual XUV photon wave packets (the pulses in the train) are converted into electron wave packets by ionization of a rare gas, and then the energy-dependent phase of the electron wave packets is measured [7]. Characterizing the electron wave packets turns out to be easier than characterizing the APT directly. The usefulness of this method crucially depends on the ability to relate the measured electron phase to the desired XUV spectral phase. The electron and XUV phases are related via the atomic dipole phase which characterizes the ionization process, and it is the calculation of this atomic phase which is our main concern in this paper.

When an attosecond XUV pulse ionizes an atom, the resulting electron wave packet (EWP) acquires an energydependent phase. This phase has two contributions, one from the spectral phase of the ionizing radiation at the excitation frequency, as well as a phase due to the atomic dipole transition which connects the bound electron to its final continuum state [10]. The total phase, the sum of these contributions, can be measured via a two-photon interference process which is illustrated in Fig. 1.

Since APTs are synthesized from a number of odd harmonics of an IR field, the electron spectrum that results from using an APT to ionize an atom is a series of peaks separated by twice the IR photon energy. When the ionization takes place in the presence of a weak IR probe field, additional IR photons can be absorbed or emitted by the electron after reaching the continuum, which results in sidebands on each of the principal peaks in the photoelectron spectrum [11-13]. Spectral interference between absorption and emission pro-

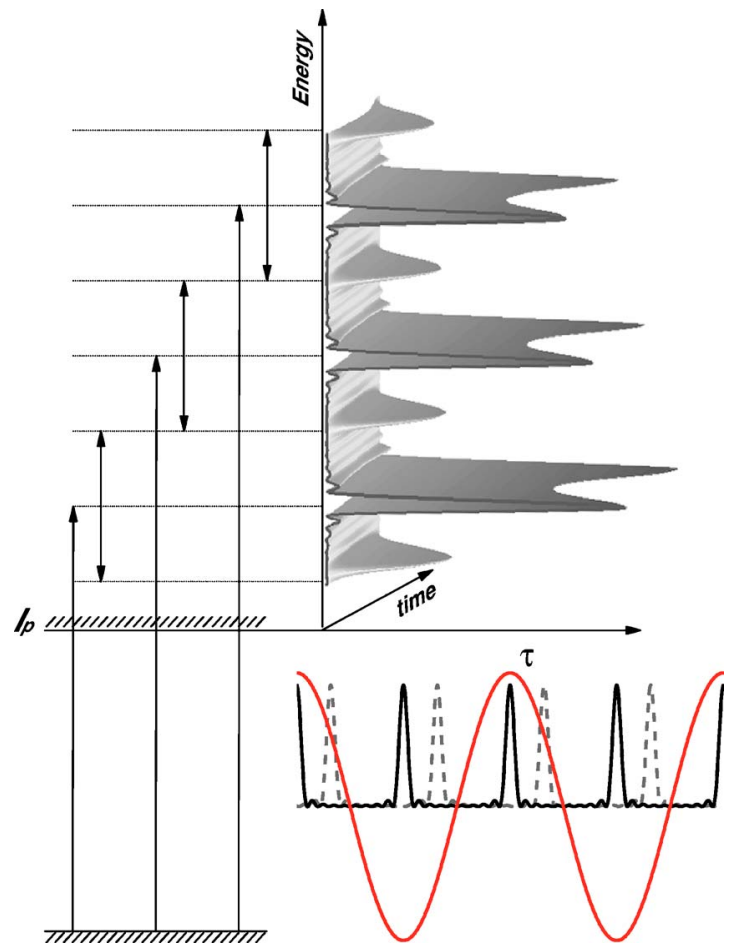

FIG. 1. (Color online) Schematic energy diagram illustrating the photoionization process. The inset depicts the APT with respect to the IR field for two different time delays. 
cesses which start from consecutive harmonics and that lead to the same final energy cause an oscillation of the sideband intensity as a function of the time delay $\tau$ between the IR and XUV pulses. The oscillatory part of the sideband intensity is proportional to $\cos \left(2 \omega_{1} \tau+\Delta_{\varphi}\right)$, where $\Delta \varphi(E)$ is the phase difference of two spectral components of the EWP which contribute to the sideband at energy $E[1,7]$. By concatenating the phase differences from several consecutive sidebands we can reconstruct (up to a trivial overall phase) the total energy-dependent phase of the EWP, $\varphi(E)$. Once the electron phase is known, the spectral phases needed for APT reconstruction can be extracted by subtracting from the total phase the phase inherited from the ionization process, the so-called atomic dipole phase. This phase has so far not been measured directly and hence must be calculated. Previously this has been done via second-order time-independent perturbation theory [14].

In this article we present a time-dependent method for calculating the atomic contributions to the properties of the electron wave packets. Our approach closely mirrors the method used experimentally to characterize APT's. Photoelectron spectra resulting from the ionization of atoms by high-order harmonics in the presence of an IR probe field are calculated for different time delays between the IR and harmonic pulses. In the experiments the electron phase is measured and the atomic dipole phase subtracted to yield the APT spectral phase. In our calculations we use perfectly phase-locked harmonics with equal amplitudes, which means that the APT spectral phase is zero. The calculated electron phase is therefore the atomic dipole phase. With this approach the electron phase can be calculated over a broad energy range from only a few time-dependent calculations and we are not limited to low IR intensities.

We study electron wave packets generated in helium, neon, and argon atoms, since these are commonly used in APT characterization experiments. For low IR intensities we find that our calculated atomic dipole phases agree well with previously published results based on perturbation theory $[1,14]$. Since our method is nonperturbative, we can also use it to investigate the dependence of the calculated atomic dipole phase on the IR probe intensity, which in an experiment must be of sufficient strength to produce a measurable interference signal. At moderate IR probe intensities, we find that the dipole phase can still be reliably determined using twophoton interference, even when higher-order processes are non-negligible.

For neon and helium the dipole phase is largest at threshold and rapidly decreases as the electron energy increases. Argon, however, is an exception to this generic behavior because of the presence of a Cooper minimum in the $3 p \rightarrow \varepsilon d$ one-photon ionization channel. We show how this structure influences the atomic phase near the minimum. We find that the Cooper minimum also significantly affects the probability for sideband generation (the probability to emit or absorb IR photons upon reaching the continuum) over a range of energies centered around the Cooper minimum. Thus we find an experimentally accessible signature of the Cooper minimum in the two-photon ionization spectra. Wellcharacterized APT's may therefore have an application in the study of continuum structures in atoms and molecules.
This article is organized as follows. In Sec. II the method of calculating the photoelectron spectra for different pulses and atoms is briefly outlined. In Sec. III the method of extracting the atomic dipole phases from the calculated spectra is presented together with the results for the different atoms. We also show how the structure of the bound-free matrix element affects the atomic dipole phase in argon. In Sec. IV we examine effects due to the IR intensity, and in Sec. V we show how the probability for sideband generation varies with photoelectron energy and how this probability is affected by structures in the continuum.

\section{METHOD}

The spectra of the electron wave packets, generated by an APT in the presence of an IR field, are calculated by solving the time-dependent Schrödinger equation (TDSE) in the single-active-electron (SAE) approximation for the mixed, multicolor field using pseudopotentials to represent the atoms $[15,16]$. The harmonics used to produce the APT are perfectly phase locked, and the phase of the electron wave packets therefore corresponds directly to the atomic dipole phase. A trapezoidal envelope has been used to model the multicolored pulse with an envelope given by

$$
\begin{gathered}
F_{q}(t)=F_{q, \text { max }}\left(t / T_{1}\right), \quad 0 \leqslant t<T_{1}, \\
F_{q}(t)=F_{q, \max }, \quad T_{1} \leqslant t<6 T_{1}, \\
F_{q}(t)=F_{q, \max }\left[7-\left(t / T_{1}\right)\right], \quad 6 T_{1} \leqslant t \leqslant 7 T_{1},
\end{gathered}
$$

where $T_{1}$ is the IR period and $q$ is the harmonic order $(q$ $=1$ for the IR pulse). The envelopes of the IR and harmonic pulses are overlapped, and the time delay $\tau$ between the pulses is then added to the harmonic part of the total electric field:

$$
E(t)=E_{1}(t)+\sum_{q} E_{q}(t+\tau),
$$

where the IR field is written as

$$
E_{1}(t)=F_{1}(t) \sin \left(\omega_{1} t\right)
$$

and the individual harmonics as

$$
\begin{aligned}
E_{q}(t+\tau) & =F_{q}(t) \sin \left[q \omega_{1} t+q \phi+\varphi_{q}\right] \\
& =F_{q}(t) \sin \left[q \omega_{1}(t+\tau)+\varphi_{q}\right],
\end{aligned}
$$

where $\omega_{1}$ is the IR frequency, $\tau=(\phi / 2 \pi) T_{1}$ is the time delay of the APT with respect to the IR field, and $\varphi_{q}$ is the phase of the $q$ th harmonic (usually set to zero for all $q$ ). The time delay is added to the APT rather than the IR field in order to decrease numerical errors arising from the one-cycle turn-on of the IR field.

Depending on the ground-state configuration, different ionization paths are possible for the atoms studied in this article, as illustrated in Fig. 2. The spin-orbit coupling for argon and neon is relatively minor and therefore ignored in the model, which results in four equivalent electrons with $m=|1|$ and two equivalent electrons with $m=0$. The total 


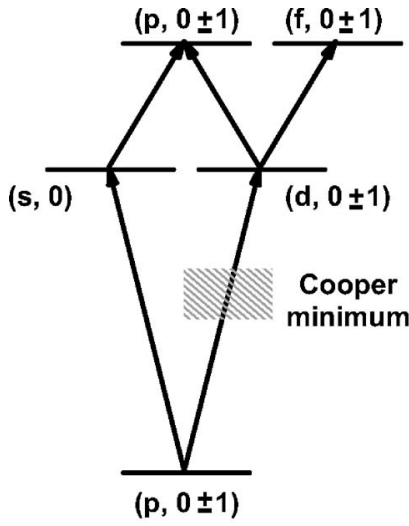

$\mathrm{Ne} \& \mathrm{Ar}$

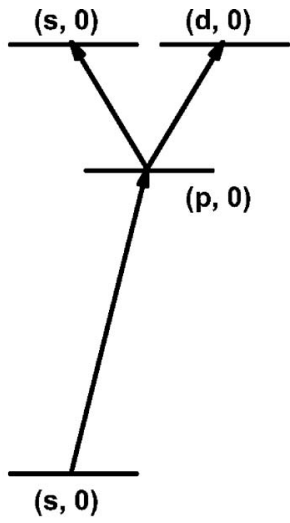

$\mathrm{He}$

FIG. 2. Different possible two-photon ionization paths for the atoms used in this article. For argon there is a Cooper minimum in the $p \rightarrow d$ one-photon ionization channel, indicated by the gray box.

photoelectron spectra are calculated by adding together the contributions from the different states weighted by the number of electrons.

The one-photon ionization cross section $\sigma_{n l}$ depends on the radial overlap between the ground-state wave function and the final continuum wave function:

$$
\sigma_{n l}(\epsilon) \propto\left|\int_{0}^{\infty} P_{n l}(r) r P_{\epsilon, l \pm 1}(r) d r\right|^{2},
$$

where $P_{n l}(r)$ and $P_{\epsilon, l \pm 1}(r)$ are the ground-state and continuum-state radial wave functions. When the final state is in the continuum the dipole matrix element [which is the radial integral in Eq. (5)] is complex and can be described as $\mu_{\epsilon g} e^{i \varphi^{a t}}$, where $\mu_{\epsilon g}$ is the norm of the ground-state-tocontinuum-dipole-matrix element and $\varphi^{a t}$ the atomic phase. If the dipole matrix element is zero for a particular energy, this yields a Cooper minimum in the cross section [17]. The effect of a Cooper minimum on the one-photon ionization cross section is well known. In this article we show that it also affects the two-photon ionization spectra.

The pseudopotentials used to represent the argon need to be carefully chosen in order to accurately take the Cooper minimum in the $3 p \rightarrow \varepsilon d$ one-photon ionization channel into account. As with helium and neon, the argon potentials are calculated using a Hartree-Fock method [16] and optimized so that the different bound-state energy levels are correctly described. This procedure, however, does not automatically ensure that the position of the Cooper minimum is correct. The argon potential can be adjusted to take account of the minimum by introducing a phase shift of the continuum $d$ states. This is achieved by introducing a small change $\delta V$ in the $d$ potential close to the core. When changing the potential the remaining atomic properties should be affected as little as possible and we therefore introduce the following constraints on $\delta V$.

(i) The Cooper minimum should be correctly positioned.

(ii) $\left\langle d_{0}|\delta V| d_{0}\right\rangle=0$, which means that there should be no shift in the lowest $d$-state energy.
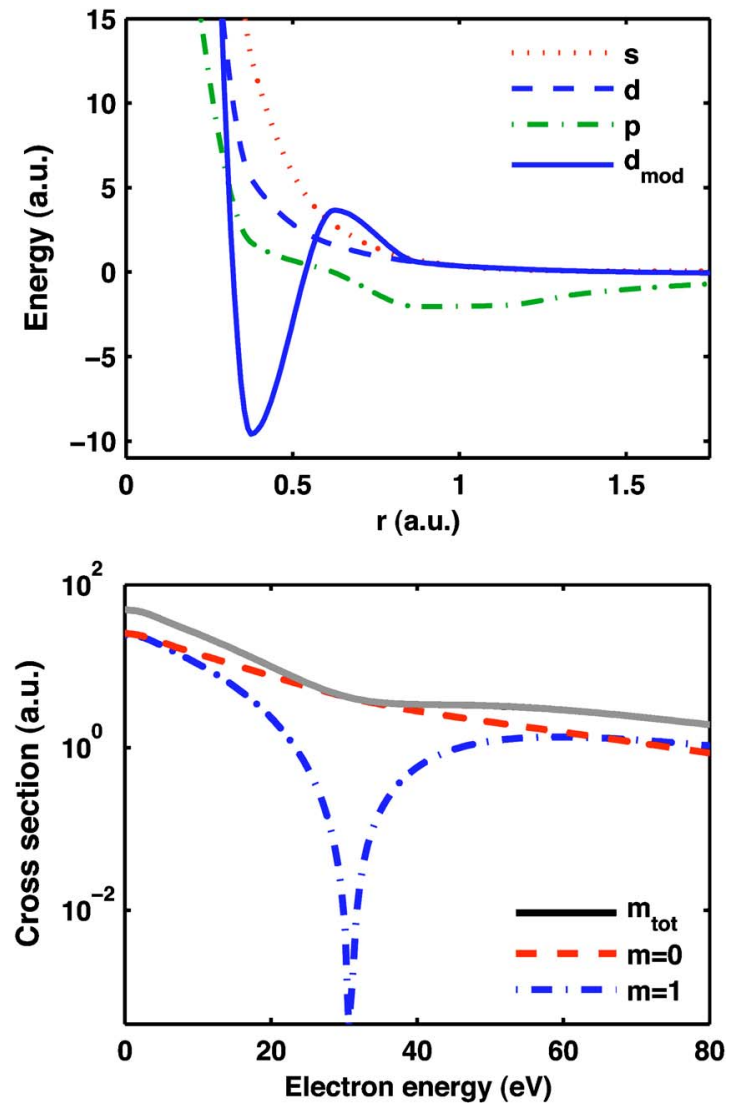

FIG. 3. (Color online) (Top) The potentials used to represent the argon atom with the centrifugal potential included. The red (dotted) line is the $s$ potential, the green (dash-dotted) line the $p$ potential, and the blue line for the $d$ potentials. The dashed blue line corresponds to the original $d$ potential whereas the solid blue line is the potential corrected in order to position the Cooper minimum at the right energy (see the text for details). (Bottom) The ionization cross section for Ar as a function of photoelectron energy is shown. The total signal (grey solid line) is dominated by contributions from $m$ $=0$ (red dashed line), and the Cooper minimum (at approximately $30 \mathrm{eV}$ ) in $m=1$ (blue dash-dotted line) therefore hardly effects the total signal.

(iii) $\int \delta V \cdot r^{2} d r=0$, which means that there should be no change in the low energy scattering strengths.

The potentials in argon are defined on a grid, where the first grid point is located at 0.125 atomic units (a.u.) and the grid spacing is 0.25 a.u. In order to simultaneously fulfill the three constraints, the $d$ potential was modified at the three grid points closest to the core. The pseudopotentials used to represent argon are shown in Fig. 3 together with the cross sections for the different $m$ channels.

\section{CALCULATING THE ATOMIC PHASES}

To extract $\Delta \varphi$ from the calculated photoelectron spectra we measure the strength of each sideband as a function of $\tau$ and fit the results to the following function:

$$
f(\phi)=\cos \left(2 \omega_{1} \tau+\pi+\Delta \varphi\right)=\cos (2 \phi+\pi+\Delta \varphi) .
$$

The additional $\pi$ is introduced because we have defined our IR field as $\sin \left(\omega_{1} t\right)$, as opposed to the cosine convention used 


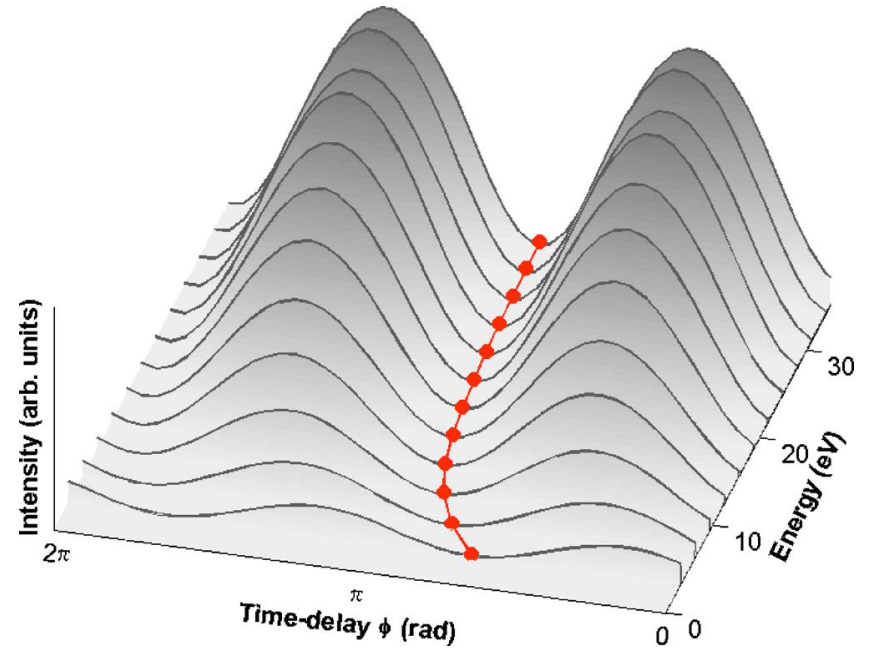

FIG. 4. (Color online) Partial photoelectron spectra in the $m$ $=0, \ell=1$ continuum state in neon as a function of time delay between the pulses; the IR intensity used in the calculation was $I_{I R}$ $=1 \times 10^{11} \mathrm{~W} / \mathrm{cm}^{2}$. This state is only populated by sidebands. The symbols mark the positions where the sideband intensity is minimized (proportional to the difference in atomic phases).

in Ref. [1]. Note that the sideband intensity is maximized when the attosecond pulses and the IR field maximum coincide, and that the sidebands oscillate with a frequency of $2 \omega_{1}$. It is therefore sufficient to vary $\phi$ between 0 and $\pi$ to recover a complete sideband oscillation with a maximum approximately in the center. Alternatively $\Delta \varphi$ can be found by finding the minimum Eq. (6) since

$$
\begin{gathered}
\frac{\partial f\left(\phi_{0}\right)}{\partial \phi}=2 \sin \left(2 \phi_{0}+\pi+\Delta \varphi\right)=0 \\
\Rightarrow \Delta \varphi=-2 \phi_{0},
\end{gathered}
$$

as illustrated in Fig. 4. The method to calculate $\Delta \varphi$ has been validated by adding known phase differences between the harmonics before calculating the photoelectron spectra. Using the method described above, the added phases were correctly retrieved after subtracting the relative atomic dipole phases.

The photoelectron spectra are calculated for the different final states separately and added together before the total atomic dipole phase is extracted. The total phase is, of course, the most interesting and experimentally accessible phase, but further insight into the origin of the atomic dipole phase can be gained if the different final states are analyzed separately. In Fig. 5 the calculated atomic dipole phases in helium, neon, and argon are shown. Both the total phases and the individual results for the different final states are shown. The atomic dipole phase in helium, shown in Figs. 5(a) and 5(b), has previously been calculated using perturbation theory [3] and our results are in excellent agreement with those data. Furthermore, it can be seen from Fig. 5(a) that the two possible final states in helium have very similar phases, which is expected since there is only one possible onephoton ionization path.
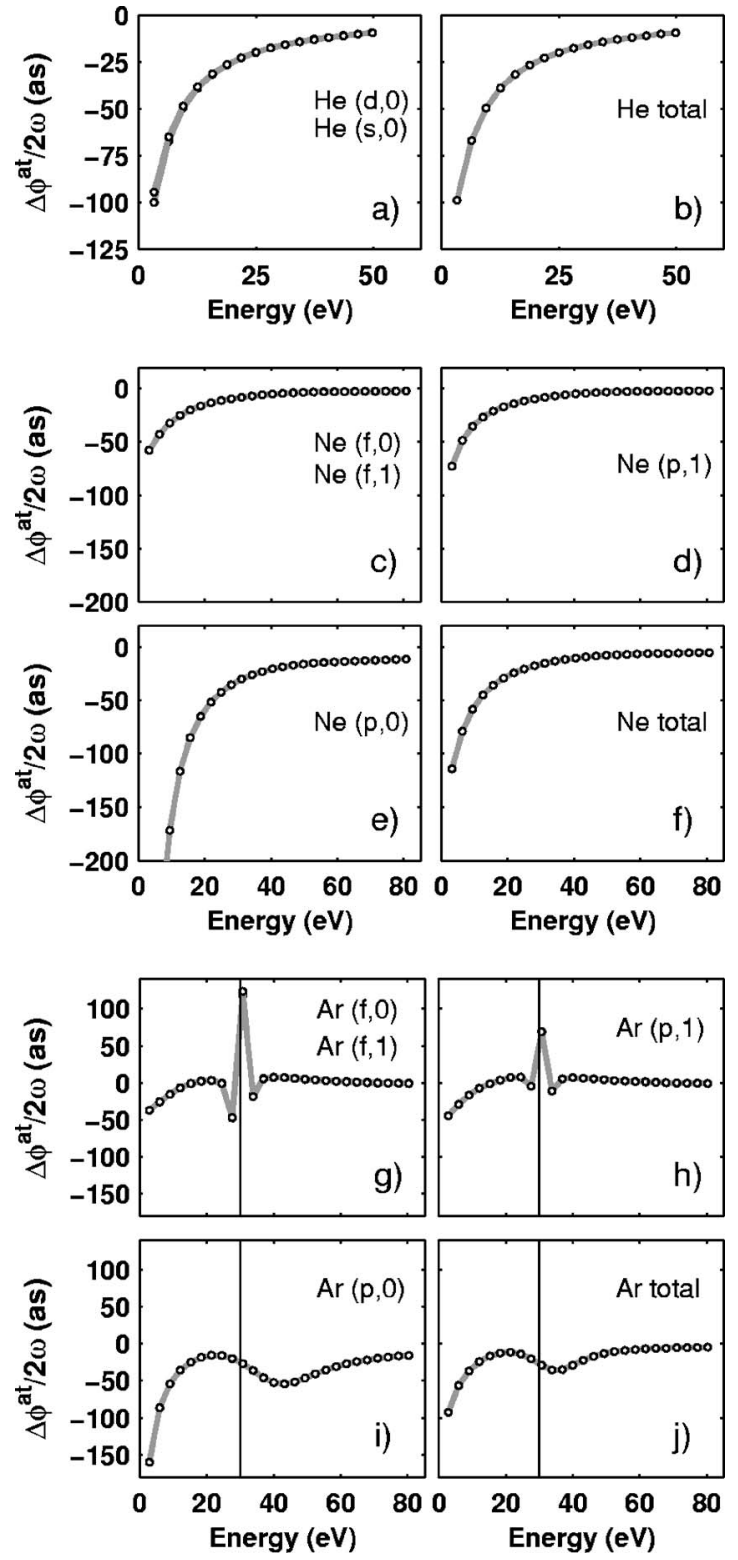

FIG. 5. The calculated $\Delta \varphi^{a t}$ for $\mathrm{He}$ (a), (b), $\mathrm{Ne}$ (c)-(f), and $\mathrm{Ar}$ (g)-(j) as a function of final electron energy. Helium: $\Delta \varphi^{a t}$ for the two different final states $[(d, 0)$ and $(s, 0) ;]$ are presented in (a) and the total $\Delta \varphi^{a t}$ is presented in (b). $\Delta \varphi^{a t}$ for the different final states in neon are presented in (c) $(f, 0)$ and $(f, 1),(\mathrm{d})(p, 1),(\mathrm{e})(p, 0)$, and (f) the total $\Delta \varphi^{a t} . \Delta \varphi^{a t}$ for the different final states in argon are presented in $(\mathrm{g})(f, 0)$ and $(f, 1),(\mathrm{h})(p, 1),(\mathrm{i})(p, 0)$, and $(\mathrm{j})$ the total $\Delta \varphi^{a t}$. For more details see the text.

For neon, with several possible ionization paths, the results are shown in Figs. 5(c)-5(f) (the different possible paths are presented in Fig. 2). It is interesting to note here that the three final states [with $(l, m)=(f, 0),(f, 1)$, and $(p, 1)]$ that are reached through the same one-photon ionization channel have very similar phases. In the fourth state [with $(l, m)=(p, 0)$ ], which can be reached through two different one-photon ionization paths, the absolute value of the atomic dipole phase is significantly increased. This increase can either be caused by interference between the two paths or simply because the atomic dipole phase of the $s$ state is 
greater than that of the $d$ state. To find the cause for the increase we have performed calculations for neon where we artificially close the ionization path through the $d$ state in the calculations. These calculations indicate that the main contribution to $\Delta \varphi^{a t}$ in the $(p, 0)$ state comes from the ionization through the $s$ state.

For argon [Figs. $5(\mathrm{~g})-5(\mathrm{j})]$ the effect of the Cooper minimum is striking. The three final states that can only be reached through the $n p \rightarrow \varepsilon d$ channel [Figs. 5(g) and 5(h)] nicely follow the generic structure except for the position where there is a harmonic on either side of the Cooper minimum in which case there is clearly a phase jump. The phase jump originates from the change of sign of the dipole matrix element [Eq. (5)] when passing the Cooper minimum. The change of sign can equally well be described as an additional phase term $e^{i \pi}$ added to the dipole matrix elements on either side of the Cooper minimum. With one harmonic on either side of the Cooper minimum this induces a $\pi$ phase shift. In the final state $(p, 0)$ that can be reached through two different paths, the structure of $\Delta \varphi^{a t}$ deviates from the generic structure over a broad energy range centered around the Cooper minimum. This deviation is a result of interference between the two paths, which have different amplitudes and phases. In addition to the phase jump in the $(p \rightarrow d)$ path there may also be an absolute phase difference between the two paths, which is not accessible with the method used, but will affect the resulting shape of the relative phase curve. At high energies $\Delta \varphi^{a t}$ again shows the generic behavior discussed in the Introduction, approaching zero even in the $(p, 0)$ final state.

We have conducted a more detailed study of argon with the emphasis on the region around the Cooper minimum using longer pulses (1-10-1 cycles) in order to get more welldefined spectral structure and we have tuned the wavelength so that one sideband is positioned exactly on the Cooper minimum. In Fig. 6 the modulation of this sideband is shown for the $m=0$ and $m=1$ final states. With $m=1$ all possible ionization channels have the Cooper minimum and the oscillation is clearly out of phase with that of $m=0$ curve, which is dominated by the channel without the Cooper minimum, due to the significantly higher one-photon absorption cross section in that channel. This shows that the phase jump is $\pi$ exactly at the Cooper minimum.

\section{INTENSITY EFFECTS}

Attosecond pulse characterization experiments are frequently performed in a moderate intensity regime in order to achieve a sufficiently strong sideband signal. At these IR intensities it is not sufficient to use perturbation theory to predict the experimental outcome. The method presented in this article to calculate the atomic phases by solving the TDSE is, however, nonperturbative, which enables us to investigate what effect the IR intensity may have on the resulting photoelectron spectra. The time-dependent sideband modulation is effected by the IR intensity in two ways: (i) there is an increased amount of electrons transferred to the sidebands which leads to depletion of the main peak and to the production of higher-order sidebands, and (ii) the ionization process is modified by the strong field, which may alter

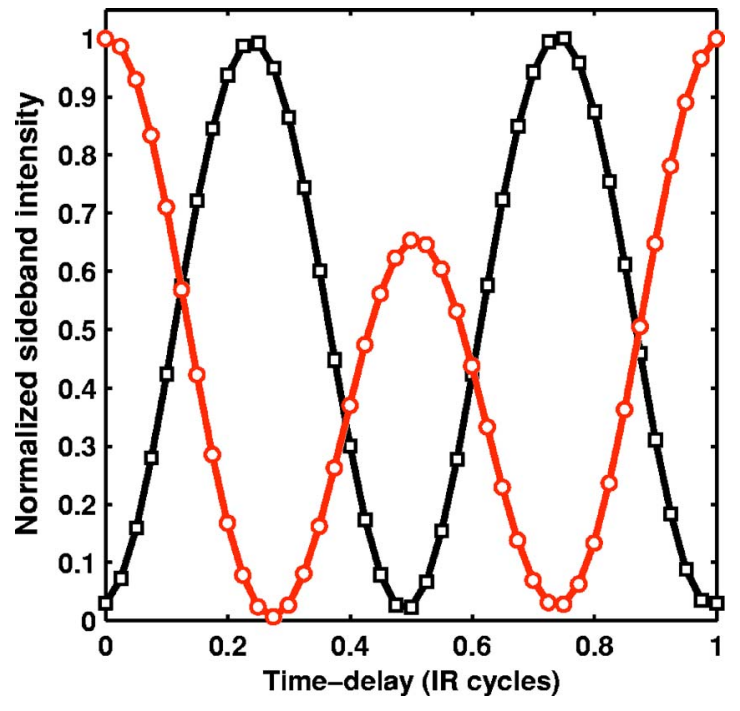

FIG. 6. (Color online) Oscillation of the sideband positioned on the Cooper minimum in argon as a function of time delay. The (black) curve with squares is calculated for $m=0$, whereas the (red) curve with circles is calculated for $m=1$, in which case the only available ionization path is the one with the Cooper minimum. The two oscillations are clearly out of phase.

the atomic dipole phase. An increasing amount of electrons transferred to the sidebands results in a deviation from the sinusoidal time dependence of the sideband signal, whereas any effect the IR intensity may have on the atomic dipole phase will yield a temporal shift of the sideband modulation.

We find that when increasing the IR intensity the changes to the sideband modulation are, by far, dominated by the increased transfer of electrons from the main peaks to the sidebands. Already at IR intensities close to $1 \times 10^{11} \mathrm{~W} / \mathrm{cm}^{2}$ (an intensity frequently used in experiments), the shape of the sideband population as a function of time delay is distorted due to depletion of the principal peak and due to interference between higher-order sidebands. Since the probability to transfer electrons from the principal peaks to the sidebands increases with increasing electron energy (see next section), sidebands at higher photoelectron energies will be more distorted compared to those generated at lower energies, for a given IR intensity. Even when the sideband modulation deviates from a sinusoidal shape, however, we find that the position of the minimum remains unaffected [see Eq. (7)], which means that the phases can still be extracted.

In order to study the effect that the IR intensity may have on the atomic dipole phase, it has first to be disentangled from the more pronounced distortions that come from the increased transfer of electrons to the sidebands. We do this by limiting the XUV field to include only two harmonics (15 and 17) and calculate the retrieved phase in argon for different IR intensities. We find that the relative phase is in fact affected by the IR intensity, and that the magnitude of $\Delta \varphi^{a t}$ increases as the square of the IR intensity (see Fig. 7).

Experimentally, problems arising from distortions of the sideband modulation are easily avoided by reducing the IR intensity so that the strengths of the sidebands never exceed 


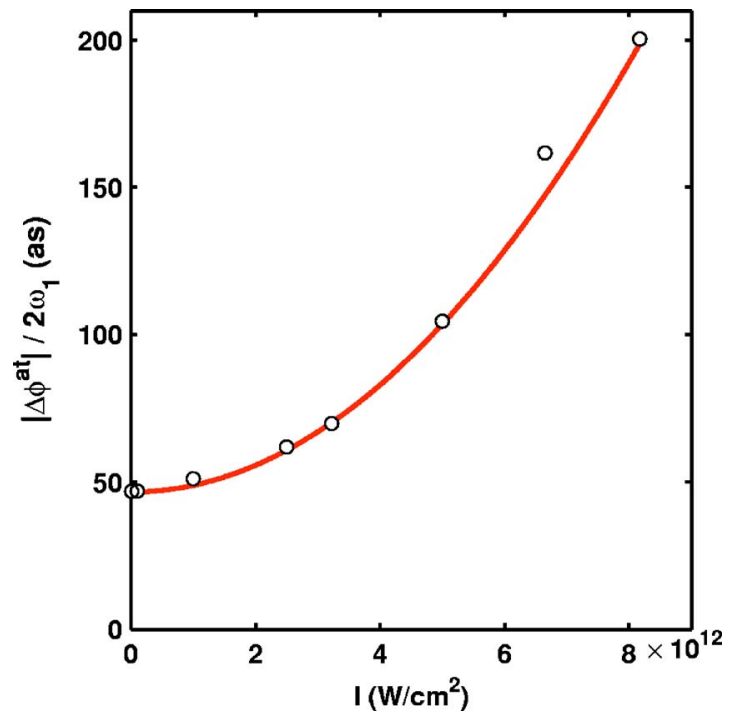

FIG. 7. (Color online) Relative atomic phase as a function of IR intensity calculated using only two harmonics (15 and 17) to ionize $\operatorname{argon}(m=0)$.

$\sim 50 \%$ of the strengths of the neighboring principal peaks, thereby reducing the intensity effects to acceptable levels. The phases of the electron wave packets are usually retrieved by taking the Fourier transform of the sideband signal and identifying the phase of the $2 \omega$ oscillation. This procedure will yield the correct value of the electron phase even when the sidebands are slightly distorted since higher-frequency components will be filtered out. The proposed constraint on the IR intensity will also ensure that the effect on the atomic phase is negligible, since the relative strength of the sidebands exceeds $50 \%$ for intensities well below what is needed to see a change in $\Delta \varphi^{a t}$. This type of APT measurement is therefore very robust against intensity fluctuations in the probe pulses.

\section{RELATIVE CROSS-SECTIONS}

The strengths of the sidebands in a two-color experiment such as we have been discussing are, of course, related to the strengths of the principal electron peaks. They also depend, however, on the final electron energy. In the absence of a structure in the ground-state-to-continuum-dipole-matrix element, the probability for the electron to emit or absorb additional IR photons increases linearly with increasing final energy [18]. Sidebands originating from absorbing a photon are therefore usually stronger than the sidebands generated by emitting a photon. Structures in the continuum may, however, alter this trend. In this section we show how the probability to generate sidebands is affected by a Cooper minimum in one of the ionization paths.

We calculate the probability to generate the sidebands by comparing the strength of the sideband with that of the unperturbed principal photoelectron peak. The calculations are done with low IR intensities in order to avoid depletion of the main peak and to avoid the generation of higher-order sidebands. If only one harmonic is used to ionize the atom,

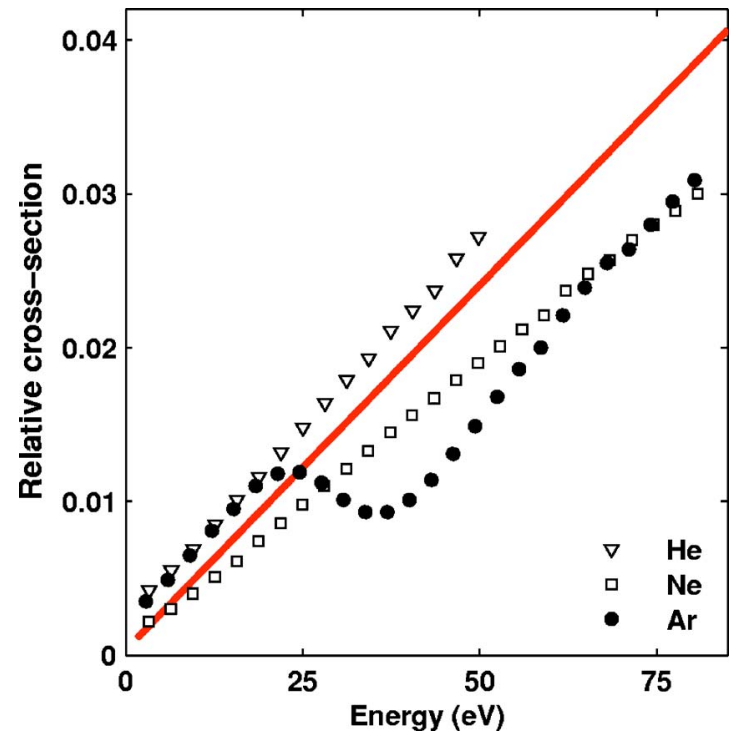

FIG. 8. (Color online) Relative cross sections as a function of photoelectron energy for helium $(\nabla)$, neon $(\square)$, and argon $(\bullet)$. The (red) solid line is calculated using perturbation theory.

the relative cross section for the first-order sidebands is defined as

$$
\begin{gathered}
\mathcal{R}_{\text {up }}(E)=\frac{S_{q+1}}{S_{q}}, \\
\mathcal{R}_{\text {down }}(E)=\frac{S_{q-1}}{S_{q}},
\end{gathered}
$$

where $\mathcal{R}_{u p}(E)\left[\mathcal{R}_{\text {down }}(E)\right]$ is the probability of absorbing [emitting] an IR photon in addition to the absorption of an XUV photon with the energy $E=q \hbar \omega_{1}$. In most experimental cases, however, each sideband is generated from two consecutive harmonics and we therefore define an average relative cross section as

$$
\langle\mathcal{R}(E)\rangle=\frac{S_{q+1}}{\frac{1}{2}\left(S_{q}+S_{q+2}\right)} .
$$

In this case the signal has to be averaged over delays spanning at least half an IR cycle or effects of the relative harmonic-atomic phase may affect the results. We have compared the results obtained using one XUV field with the case of several XUV fields and find that they agree very well even though the probability to absorb one IR photon is slightly higher than the probability to emit an IR photon.

In Fig. 8 the calculated values of $\mathcal{R}$ as a function of electron energy for helium, neon, and argon are shown, together with the values expected for a flat continuum [18]. For He and $\mathrm{Ne}, \mathcal{R}$ increases linearly, with slopes close to the prediction based on a flat continuum. For Ar, however, there is clearly a deviation from this trend. In the region around $30 \mathrm{eV}, \mathcal{R}$ actually decreases. The anomalous behavior is centered around the Cooper minimum, and we attribute the deviation from a straight line to the presence of a Cooper mini- 


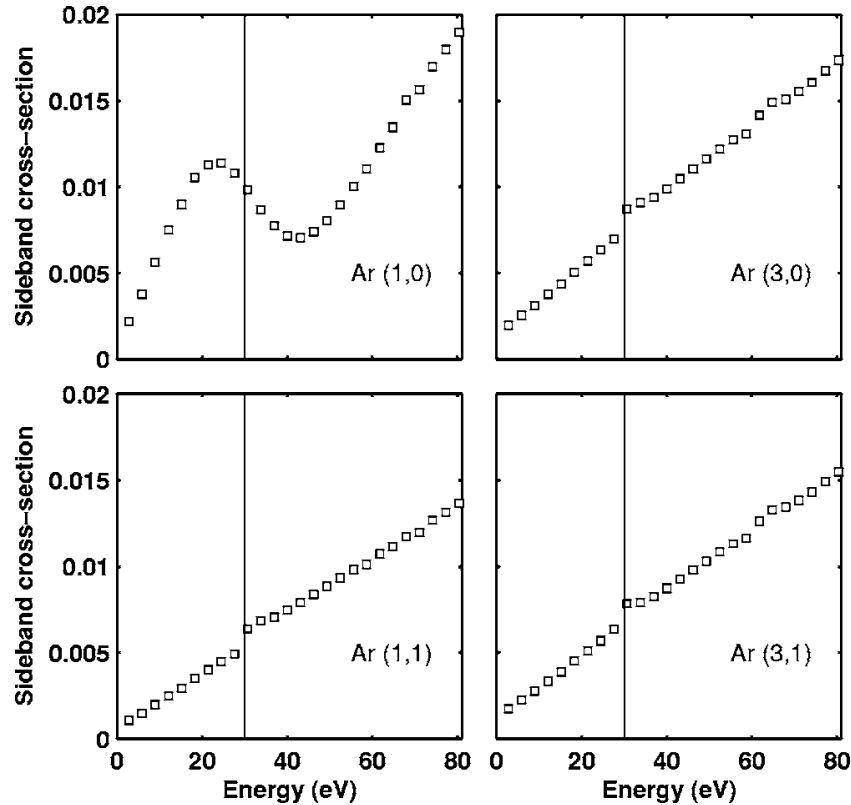

FIG. 9. Relative cross sections in argon as a function of photoelectron energy. The final quantum states are presented in brackets in each panel. The lines mark the position of the Cooper minimum.

mum in the $3 p \rightarrow \varepsilon d$ channel. By analyzing the different possible final states individually (Fig. 9) it is clear that the anomalous behavior is only present in the final state that is reached through two different ionization paths. Interference between the two different paths that lead to the same final state results in a delicate balance that is strongly affected by the presence of the Cooper minimum.

Experimentally $\mathcal{R}$ is an easily accessible parameter, and it is therefore promising that it is so clearly affected by atomic structures. Since $\mathcal{R}$ is measured by comparing the strength of the sidebands to that of the neighboring principal peaks, the measurement will be very robust against experimental errors. The energy dependence of the detection will, for instance, cancel out since it is a relative measurement. Fluctuations are, for the same reason, not of major concern either. The effect is presumably even larger in atoms that have an $s$ ground-state configuration and a Cooper minimum in the $n s \rightarrow \varepsilon p$ ionization channel (the alkali-metal atoms for instants).

\section{CONCLUSION}

In this article we have presented a nonperturbative, timedependent method to calculate the intrinsic properties of electron wave packets ionized by APT's. The method has been used to calculate the experimentally important atomic dipole phases of helium, neon, and argon. It has further been used to study what influence a Cooper minimum in one of the ionization channels has on the properties of the ionized wave packets. The Cooper minimum is found to introduce a phase jump in the atomic dipole phase, and it also affects the relative sideband strengths, thereby producing an experimentally easily accessible signature of the Cooper minimum in the two-photon ionization spectra. Since the method is nonperturbative, we have been able to study intensity effects and found that the experimental method used to characterize APT's is rather insensitive to variations in the IR intensity in the intensity regime where most experiments of this type are performed $\left(I_{I R}\right.$ is typically less than $\left.10^{11} \mathrm{~W} / \mathrm{cm}^{2}\right)$.

\section{ACKNOWLEDGMENTS}

J.M. and K.S. acknowledge the support of the National Science Foundation through Grant No. PHY-0401625, and M.G. acknowledges the support of the Louisiana Board of Regents through Grant No. LEQSF(2004-07)-RD-A-09.
[1] P. M. Paul et al., Science 292, 1689 (2001).

[2] S. A. Aseyev, Y. Ni, L. J. Frasinski, H. G. Muller, and M. J. J. Vrakking, Phys. Rev. Lett. 91, 223902 (2003).

[3] Y. Mairesse et al., Science 302, 1540 (2003).

[4] P. Tzallas, D. Charalambidis, N. A. Papadogiannis, K. Witte, and G. D. Tsakiris, Nature (London) 426, 267 (2003).

[5] L. C. Dinu, H. G. Muller, S. Kazamias, G. Mullot, F. Augé, P. Balcou, P. M. Paul, M. Kovačev, P. Breger, and P. Agostini, Phys. Rev. Lett. 91, 063901 (2003).

[6] R. López et al., Phys. Rev. Lett. 94, 033001 (2005).

[7] H. G. Muller, Appl. Phys. B: Lasers Opt. 74, S17 (2002).

[8] P. Agostini and L. F. DiMauro, Rep. Prog. Phys. 67, 813 (2004).

[9] M. Ferray et al., J. Phys. B 21, L31 (1988).
[10] V. Véniard, R. Taïeb, and A. Maquet, Phys. Rev. A 54, 721 (1996).

[11] V. Véniard, R. Taïeb, and A. Maquet, Phys. Rev. Lett. 74, 4161 (1995).

[12] T. E. Glover, R. W. Schoenlein, A. H. Chin, and C. V. Shank, Phys. Rev. Lett. 76, 2468 (1996).

[13] J. M. Schins et al., J. Opt. Soc. Am. B 13, 197 (1996).

[14] E. S. Toma and H. G. Muller, J. Phys. B 35, 3435 (2002).

[15] K. J. Schafer, Comput. Phys. Commun. 63, 427 (1991).

[16] K. C. Kulander and T. N. Rescigno, Comput. Phys. Commun. 63, 523 (1991).

[17] J. W. Cooper, Phys. Rev. 128, 681 (1962).

[18] H. G. Muller, H. B. van Linden van den Heuvell, and M. J. van der Wiel, J. Phys. B 19, L733 (1986). 